\title{
Research on Evaluation Method of Jamming Effect on ISAR Using Wavelet Moment
}

\author{
Huang Jianchong ${ }^{1,}$, CuiRui ${ }^{2, b}$ ZhuJing $^{3, c}$ \\ ${ }^{1}$ Electronic Engineering Institute, Hefei, China \\ ${ }^{2}$ Electronic Engineering Institute, Hefei, China \\ ${ }^{2}$ Army Office Academy, Hefei, China \\ a2654249794@qq.com, ${ }^{\text {b } 21047798 @ q q . c o m ~}{ }^{\text {c }}$ zhujing699@sohu.com
}

Keywords: Jamming effect; wavelet Moment; evaluation method; ISAR

\begin{abstract}
ISAR is a hot and difficult issue in the field of electronic counter measures (ECM). Traditional evaluate methods are unsuitable to ISAR because of its new system; a new evaluation method of barrage jamming on ISAR is given according to the principle of radar jamming and the theory of target recognition. The wavelet moment is chosen as an index to evaluate the jamming effect through calculating the difference of two picture's wavelet Moment; the simulation results of two typical noise barrage jamming modes prove the method is corrective and effective.
\end{abstract}

\section{Introduction}

With the fast development of radar technology, the Inverse Synthetic Aperture Radar (ISAR) has been a most important military reconnaissance way, which can be widely used for target classification, recognition and identification in military field [1]. As a main part of Electronic Countermeasures (ECM), Evaluation method of jamming effect on ISAR attracts much more attention.

Currently, the few open report on the evaluation method of ISAR can be seen in the word because its secrecy. The evaluation method can be classed as two sorts: subjective and impersonal method. The Equivalent Number of Looks 、Correlation Coefficient and Kurtosis are used to evaluate the jamming effect. There is no a suit of impersonal and quantificational standard to evaluate the jamming effect on ISAR. Traditional methods used to evaluate the jamming effect of common radar are unsuitable to the ISAR. By comparing the change of image wavelet Moment, a novel evaluation method of jamming effect on ISAR is presented in the paper, which can reflect the impact on the imaging quality by the jamming.

\section{The description of wavelet Moment}

The military use of ISAR is to recognize target, and the picture is the final product of ISAR. The aim of jamming ISAR is to destroy target recognition. The target recognition probability lies on the choice of characters mostly. The following characters are often used to recognize target: geometry moment, figure characters, airscrew modulated characters. Because of having the ability of circumrotation fixity, the moment character is one of the most important characters for ISAR target recognition. [2]

the moment characters used in picture recognition are HU moment 、Zernike moment and wavelet moment etc. Compared with HU moment, wavelet moment has many merits, which is orthogonal moment. It can construct any high-rank moment and also can keep the original scale, translation and rotation invariance. So wavelet moment is introduced to evaluate the jamming effect of ISAR. [3]

One two-dimension picture $f(x, y)$ is given, and then the wavelet moment can be determined as following formula: 


$$
M_{p q}=\iint x^{p} y^{p} f(x, y) d x d y
$$

For $x=r \cos (\theta), y=r \sin (\theta),{ }^{M}{ }_{p q}$ can be changed as following formula:

$$
F_{p q}=\iint f(r, \theta) g_{p}(r) e^{j q \theta} r d r d \theta
$$

Where, $g_{p}(r)$ is the radial component of transform kernel, $e^{j q \theta}$ is the angular component.

$$
\text { If } S_{q}(r)=\int f(r, \theta) e^{j q \theta} d \theta \text {, then the above formula can be changed as: }
$$

$$
F_{p q}=\int s_{q}(r) g_{p}(r) r d r
$$

The image is destroyed by noise, so the wavelet moment of image is changed. As a result, the recognition probabilities fall. The wavelet moment is calculated respectively in the different jamming mode, and then the difference $\Delta F$ between jammed image and origin image are calculated. With the same JSR, if $\Delta F$ is bigger, then the jamming effect is better.

\section{Simulation of jamming effect}

The ideal plan point target model is adopted to carry out the simulation of barrage jamming on ISAR in the paper. The parameters of system are represented as follows: Signal carrier frequency f0 $=11 \mathrm{GHz}$, bandwidth $\mathrm{B}=300 \mathrm{MHz}$, Pulse-width $\tau=3 u s, P R F=250 \mathrm{HZ}$, the radial velocity of plan $\mathrm{v}=20 \mathrm{~m} / \mathrm{s}$. Noise AM jamming and noise FM jamming are carried out in the paper. The signal of noise AM can be described as[4]-[5]:

$$
J(t)=\left(U_{0}+u_{n}(t)\right) \cos \left(2 \pi f_{0} t+\varphi\right)
$$

Where modulated noise $u_{n}(t)$ is a wide-sense stationary stochastic processes, its mean is zero and variance is $\sigma_{n}^{2}$, amplitude of carrier wave is $U_{0}$, carrier frequency is $f_{0}$, original phase of carrier wave is $\varphi$.The signal of noise FM can be expressed as:

$$
J(t)=U_{0} \cos \left[2 \pi f_{j} t+2 \pi k_{F H} \int_{0}^{t} u(\tau) d \tau+\phi\right]
$$

Where modulated noise $u(t)$ is a wide-sense stationary stochastic processes, its mean is zero and variance is $\sigma_{n}^{2}$, amplitude of carrier wave is $U_{0}$, carrier frequency is $f_{i}$, original phase of carrier wave is $\varphi$, coefficient of frequency modulation is $k_{F U}$.

Fig. 1 and Fig. 2 are the image that have been jammed in the two jamming mode with the same JSR (5dB and 10dB), when JSR is $5 \mathrm{~dB}$, from Fig.1a, we can still distinguish the plan even though it has been jammed, however the image in Fig.2a has been destroyed badly. When JSR is 10dB, both of them have been destroyed badly, but we still can see clearly that the jamming effect of noise FM is better than the effect of noise AM.

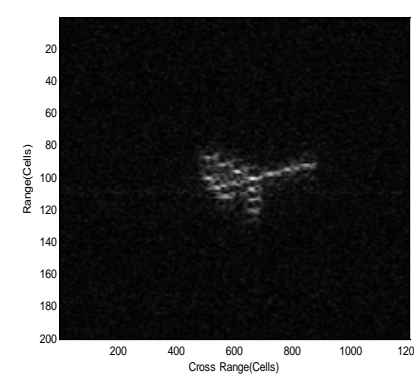

(a) $\mathrm{JSR}=5 \mathrm{~dB}$

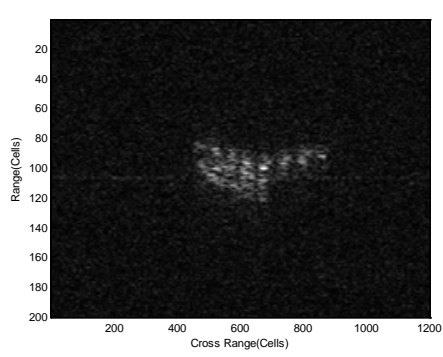

(b) $\mathrm{JSR}=10 \mathrm{~dB}$

Fig.1 ISAR image with noise AM jamming 


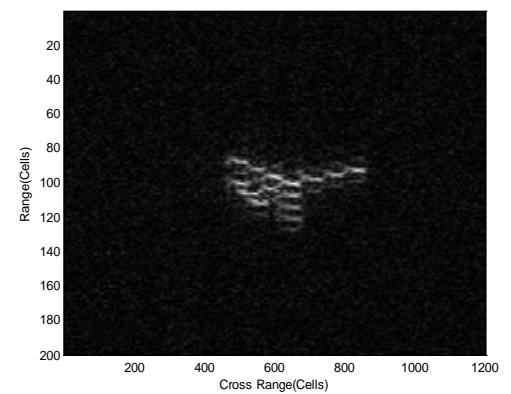

(a) $\mathrm{JSR}=5 \mathrm{~dB}$

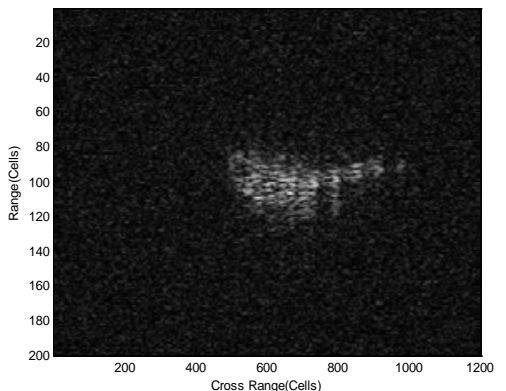

(b) $\mathrm{JSR}=10 \mathrm{~dB}$

Fig.2 ISAR image with noise FM jamming

\section{Quantitative jamming effect evaluation}

The wavelet moment difference of two images can be calculated according to formula (3). The relationship between JSR and them can be shown as Fig.3: [6]-[7]

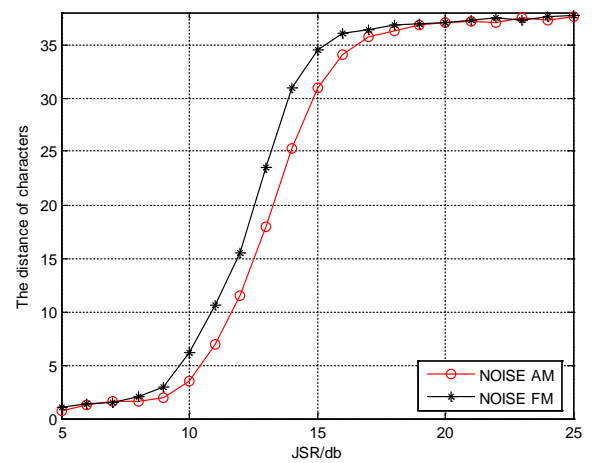

Fig.3 curve of the distance of wavelet moment

The conclusions can be got from the figure 3:

1. With the increasing of JSR and the image is destroyed more badly. The curve of the distance of wavelet moment rise clearly.

2. With the same JSR, the distance of wavelet moment that jammed by noise FM is bigger than that jammed by noise AM. It proves that the jamming effect of noise FM is better than noise AM, which is consistent with Fig 2.

3. Two curves get together when JSR is bigger than $15 \mathrm{~dB}$, which mean the image have been jammed badly and the noise have made evaluation criterion invalidated.

\section{Conclusions}

The quality of image becomes worse and worse with the increasing of jamming power, so it is difficult to recognize target. At the same time, the wavelet moment changes with the jamming power. Compared with HU moment, wavelet moment is orthogonal moment; the wavelet moment is analyzed deeply and applied to evaluate the jamming effect on ISAR. Through the change of the distance of wavelet moment, the method of quantitative jamming effect evaluation on ISAR is carried out. The result of simulation has been proved corrective and effective by.

\section{References}

[1] CHEN C C. Target-Motion-Induced Radar Imaging [J]. IEEE Trans on AES.1980, 16(1):2-14.

[2] Dudani, BA, Breeding, KJ and Mc Ghee, RG. Aircraft Identification by Moment Invariants. IEEE. Trans, Compute, 1977, 26: 39-46 
[3]Yao Jun Jiang Xiao-Yu Huang Ying-Qing. comparison between Hu Moment、Zernike Moment and Wavelet Moment in Target Recognition [J]. Journal of academy of armored force engineering, 2006, 20(3):34-36

[4] LENG Chuan-hang, FU YU-Sheng, PI Yi-Ming, HOU Yin-ming. Research on simulation of interference on inverse synthetic aperture radar[J]. the technology of electronic warfare, 2005, (5) : 35-39.

[5] MIAO Yan-hong, ZHAO Guo-qing. Measurement of the jamming effect of synthetic aperture radar [J]. The technology of electronic warfare, 2004, 19 (4) : 19-25.

[6]Cui Rui, Xue Lei, Wang Bo. Evaluation method of jamming effect on ISAR Based on Equivalent number of looks[J]. Systems Engineering and Electronics.2008， 30(5):887 - 888.

[7] Li Yuan, Chen Huilian, "Evaluation method of jamming effect on ISAR Based on Correlation Coefficient,” Journal of University of Electronic Science and Technology, vol.35,no.4,Aug.2006,pp.468 -470. 